\title{
STRESS DISTRIBUTIONS IN ELASTIC AND VISCOELASTIC PLATES SUBJECTED TO SYMMETRICAL RIGID INDENTATIONS*
}

\author{
BY \\ Y. M. TSAI \\ Iowa State University
}

I. Introduction. In a previous paper [1], the static and dynamic tensile strengths of glass blocks were measured by the methods of spherical indentation and impact. That paper was concerned with the apparent dependence of the values of the fracture strength on the radius of the spherical indenter. The theory developed there critically depends upon the validity of the Hertz treatment for an elastic half space [2], [3]. In later work [4] the nature of fractures produced in glass blocks by impact was studied by measuring the fracture stress waves as a function of impact velocities. From the stress waves experimentally observed and a knowledge of wave propagation, it could be estimated that at the highest velocities of impact, fractures occurred only a few microseconds after the glass blocks were first compressed. At the instant of occurrence of fracture a thin layer of glass was stressed while the rest of the glass block was still free from traction. The physical situation would appear to be that of a thin layer of stressed material resting on a rigid foundation. It was also observed [4] that at high impact velocity large radial cracks were produced inside the material. This pattern of fractures, however, was not observed at lower impact velocities, where a relatively thicker layer of glass was stressed at the instant of fracture. This velocity effect on the patterns of fractures would lead one to consider the possible effect that the thickness of the stressed material has on the local stress distribution. It is with these considerations and with the practical applicability of the Hertz solution, for plates of finite thickness, that the present investigation is concerned.

An elastic plate of thickness $H$ which is subjected to an axisymmetrical indentation on its upper surface, and which rests on a rigid smooth foundation, is mathematically equivalent to a plate of thickness $2 H$ subjected to equal indentations on its two surfaces. In the work described here the normal contact stress on the surface of the plate is studied as a function of the radius of contact and the thickness of the plate. In terms of the same geometrical quantities the radial and circumferential surface stresses are also investigated. Furthermore, the elastic contact stress solution for the plate is extended to the linear viscoelastic solid.

II. Elastic contact stress. Consider an infinite elastic plate of thickness $H$ which is subjected to a rigid, axisymmetrical indentation on its upper surface $z=H$, but resting on a smooth, rigid foundation at $z=0$. This problem is equivalent to a plate of thickness $2 H$ subjected to the same indentations on both upper and lower surfaces. It is assumed that the shear stress vanishes on the surfaces, and that the normal contact stresses $p(r)$ are formally known functions of the radial distance $r$. In fact, $p(r)$ will be found as the solution to an integral equation. Thus the problem is axisymmetrical, and it can be solved

\footnotetext{
*Received May 4, 1968; revised version received October 17, 1939.
} 
by using the harmonic Boussinesq functions $\Phi$ and $\Psi$ [5]; i.e.,

$$
\nabla^{2} \Phi=\nabla^{2} \Psi=0 .
$$

In terms of these two functions,

$$
2 \mu U_{z}=\partial \Phi / \partial z+z \partial \Psi / \partial z-(3-4 v) \Psi
$$

and

$$
\sigma_{z z}=\partial^{2} \Phi / \partial z^{2}+z \partial^{2} \Psi / \partial z^{2}-2(1-\nu) \partial \Psi / \partial z
$$

where $U_{z}$ is the vertical displacement, and $\nu$ and $\mu$ are, respectively, Poisson's ratio and the shear modulus. Using the method of Hankel transforms [4] to solve (1) subject to the boundary conditions that $\sigma_{z r}=0$ and $\sigma_{z z}=p(r)$ for $r \leq a$, but vanishes for $r>a$ at $z= \pm H$

$$
\begin{aligned}
& \Phi=\int_{0}^{\infty} \frac{2 \bar{p}(s)[s H \cosh s H-(1-2 \nu) \sinh s H]}{s F(2 H s)} \cosh s z J_{0}(s r) d s, \\
& \bar{\psi}=-\int_{0}^{\infty} \frac{2 \bar{p}(s) \sinh s H}{F(2 H s)} \sinh s z J_{0}(s r) d s
\end{aligned}
$$

and

$2 \mu U_{z}=\int_{0}^{\infty} \frac{2 \bar{p}(s)}{F(2 H s)}\{[s H \cosh s H+2(1-\nu) \sinh s H] \sinh s z-s z \sinh s H \cosh s z\} d s$

where

$$
\begin{aligned}
F(2 s H) & =2 s H+\sinh 2 s H, \\
\bar{p}(s) & =\int_{0}^{a} p(r) r J_{0}(s r) d r,
\end{aligned}
$$

$J_{0}(x)$ is the Bessel function of the first kind of zero order, and $a$ is the radius of the area of contact. Then (4) and (5) will be used in the next section for determining stress distributions.

If the shape of the vertical displacement produced by the indenter inside the area of contact can be described by $g(r)$, we have $U_{z}=g(r)$ for $0 \leq r \leq a$ at $z=0$. If we let $z=H$ and replace $U_{z}$ by $g(r)$ in (6), we have

$$
g(r)=2 C \int_{0}^{\infty} J_{0}(s r) \frac{\sinh ^{2} s H}{F(2 H s)} d s \int_{0}^{a} p(\lambda) \lambda J_{0}(\lambda s) d \lambda \text { for } 0 \leq r \leq a
$$

where $C=(1-\nu) / \mu$. This may be considered as an integral equation for the unknown $p(r)$. We shall first examine the extreme case where the value of $H$ is large. If we let $H$ tend to infinity, the kernel function $\sinh ^{2} s H / F(2 H s)$ approaches $1 / 2$ and (9) becomes precisely the equation obtained in the half-space problem [6], [7], which can then be solved by the same method as employed in [7]. Thus the contact stress for a half-space problem is

$$
p_{H}(r)=-\frac{2}{C \pi r} \frac{\partial}{\partial r} \int_{r}^{a} \frac{\zeta d \zeta}{\left(\zeta^{2}-r^{2}\right)^{1 / 2}} \frac{\partial}{\partial \zeta} \int_{0}^{\zeta} \frac{m g(m) d m}{\left(\zeta^{2}-m^{2}\right)^{1 / 2}} .
$$

The right side of (10) clearly indicates the procedures for inverting the integral equation. 
With the condition that $p_{H}(r)$ vanishes at $r=a,(10)$ becomes the more realistic form

$$
\begin{aligned}
p_{H}(r) & =-\frac{2}{C \pi} \int_{r}^{a} \frac{1}{\left(\zeta^{2}-r^{2}\right)^{1 / 2}} \frac{\partial}{\partial \zeta} \int_{0}^{\zeta} \frac{\zeta g^{\prime}(m) d m}{\left(\zeta^{2}-m^{2}\right)^{1 / 2}} d \zeta \quad r \leq a, \\
& =\quad 0 \quad r>a,
\end{aligned}
$$

with an auxiliary equation

$$
g(0)+a \int_{0}^{a} \frac{g^{\prime}(m) d m}{\left(a^{2}-m^{2}\right)^{1 / 2}}=0 .
$$

Eq. (12), together with the following equation for the applied load

$$
P=2 \pi \int_{0}^{a} p(r) r d r
$$

can be used to determine the radius of contact $a$ and the depth of penetration $g(0)$ at the center of the contact area.

The above method of solution for the half-space can also be used to solve for the contact stress in the plate of finite thickness. To do this, we write (9) as

$$
g(r)=C \int_{0}^{\infty} J_{0}(s r) \bar{p}(s) d s-C \int_{0}^{\infty} Q(2 H s) J_{0}(s r) \bar{p}(s) d s
$$

where

$$
Q(2 H s)=\frac{1+2 H s-e^{-2 H s}}{F(2 H s)} .
$$

If we apply the same procedures as in (10) to (14) and satisfy the condition $p(a)=0$, we have the contact stress

$$
p(r)=p_{H}(r)+\int_{0}^{a} p(\lambda) \lambda K(r, \lambda) d \lambda
$$

and the subsidiary equation for determining $g(0)$

$$
g(0)+a \int_{0}^{a} \frac{g^{\prime}(m) d m}{\left(a^{2}-m^{2}\right)^{1 / 2}}+\frac{C}{2 H} \int_{0}^{\infty} Q(u) \cos \left(\frac{a u}{2 H}\right) \bar{p}\left(\frac{u}{2 H}\right) d u=0
$$

where

$$
u=2 H s
$$

and

$$
K(r, \lambda)=\frac{1}{2 \pi H^{2}} \int_{0}^{\infty} Q(u) u J_{0}\left(\frac{\lambda u}{2 H}\right) d u \int_{r}^{a} \frac{\sin (\zeta u / 2 H) d \zeta}{\left(\zeta^{2}-r^{2}\right)^{1 / 2}} .
$$

On the right-hand side of (16) the first term, which is the previous half-space solution, can be considered as a known function. However, the second term described the effect of the plate thickness in terms of the unknown $p(r)$ and vanishes as $H$ tends to infinity. Then (16) is a Fredholm integral equation of the second kind with the complicated kernel function $K(r, \lambda)$; it can be solved by many standard techniques. Indeed, it will be shown later that the method of successive approximations is an effective method of solution. 
To solve (16) the shape of the indenter must first be specified. Half-space solutions for $p_{H}(r)$ have been obtained for indenters of various simple geometrical shapes [7]. The corresponding solutions for plates of finite thickness can presumably be obtained by solving (16), but only the case of the spherical indentation will be considered here.

Consider a rigid spherical indenter of radius $R$. If the radius of the contact circle is small compared to $R$ and if the maximum depth of indentation is $\alpha$, we may write

$$
g(r)=r^{2} / 2 R-\alpha .
$$

If we substitute (20) into (11) we have the half-space solution [3], [7]

$$
\begin{aligned}
p_{H}(r) & =-4\left(a^{2}-r^{2}\right)^{1 / 2} / C \pi R . \\
p_{H}(r)=p_{H}(a x) & =-4 a\left(1-x^{2}\right)^{1 / 2} / C \pi R .
\end{aligned}
$$

If we now let $\zeta=a \eta$ and $\lambda=a y$, respectively, in (19) and (16), we may write by the method of successive approximations using (22) the solution of (16) as

$$
\begin{aligned}
p(r)=p(a x)=-\frac{4 a}{C \pi R}[ & \left(1-x^{2}\right)^{1 / 2}+\frac{2}{\pi}\left(\frac{a}{2 H}\right)^{2} \int_{0}^{1}\left(1-y^{2}\right)^{1 / 2} y d y \\
& \left.\cdot \int_{0}^{\infty} Q(u) u J_{0}\left(\frac{a}{2 H} y u\right) d u \int_{x}^{1} \frac{\sin (a / 2 H \eta \eta) d \eta}{\left(\eta^{2}-x^{2}\right)^{1 / 2}}+\cdots\right] .
\end{aligned}
$$

From (23) and (22) we may see that the ratio $p(r) / p_{H}(r)$, and the effect that the thickness has on $p(r)$, depend not on the material constant $C$, nor the radius $R$, but the geometric ratio $a / H$. These two quantities increase with increasing $a / H$ as shown in Fig. 1(a), (b). When $a / H$ is less than 0.06 the half-space solution is apparently an accurate approximation to the plate solution. If we increase $a / H$ to 0.48 , the value of the maximum contact stress in the plate is $3.5 \%$ larger than the corresponding half-space quantity. However, when $a / H$ equals 0.96 the maximum contact stress in the plate is $20 \%$ larger than that in the half-space. Thus when the value of $a$ is comparable with $H$, the plate action can not be neglected in calculating contact stresses.

In solving for the contact stresses, the singularity at $\zeta=r$ in (19) can be avoided by using the following alternative form

$$
\begin{aligned}
\int_{r}^{a} \frac{\sin (\zeta u / 2 H)}{\left(\zeta^{2}-r^{2}\right)^{1 / 2}} d \zeta=\frac{\sin (a u / 2 H)}{a} & \left(a^{2}-r^{2}\right)^{1 / 2} \\
& \quad-\int_{r}^{a} \frac{\left(\zeta^{2}-r^{2}\right)^{1 / 2}}{\zeta^{2}}\left[\frac{\zeta u}{2 H} \cos \left(\frac{\zeta u}{2 H}\right)-\sin \left(\frac{\zeta u}{2 H}\right)\right] d \zeta .
\end{aligned}
$$

The first approximation for $p(r)$ in (16) is naturally the half-space term $p_{H}(r)$. For $a / H=$ 0.06 the second approximation is almost identical with the first term. For $a / H=0.48$ and 0.96 , however, the differences between the third and fourth approximations were less than 0.3 percent. Thus the convergence of the method of successive approximations is fast, and the method is apparently a satisfactory one.

III. Stress distribution. We now examine the stress distribution produced by the surface indentation. In terms of the Boussinesq functions, we can obtain from (4) and (5) the radial stress [5] 


$$
\begin{aligned}
\sigma_{r r}= & \partial^{2} \Phi / \partial r^{2}+z \partial^{2} \Psi / \partial r^{2}-2 \sigma \partial \Psi / \partial z \\
= & 2 \int_{0}^{\infty}\left\{\left[\frac{J_{1}(r s)}{r}-s J_{0}(r s)\right][(s H \cosh H s-(1-2 \nu) \sinh s H) \cosh s z\right. \\
& \left.-s z \sinh s H \sinh s z]+2 \nu J_{0}(r s) s \sinh s z \cosh s z\right\} \frac{\bar{p}(s)}{F(2 H s)} d s
\end{aligned}
$$

and the circumferential stress

$$
\begin{aligned}
\sigma_{\theta \theta}= & -2 \int_{0}^{\infty}\left\{\frac{J_{1}(s r)}{r}[(s H \cosh s H-(1-2 \nu) \sinh s H) \cosh s z\right. \\
& \left.-s z \sinh s H \sinh s z]-2 \nu J_{0}(r s) s \sinh s H \cosh s z\right\} \frac{\bar{p}(s)}{F(2 H s)} d s .
\end{aligned}
$$

If we let $z$ be equal to $H$ in (25), we have the radial stress in the surface of indentation. Thus at $z=H$

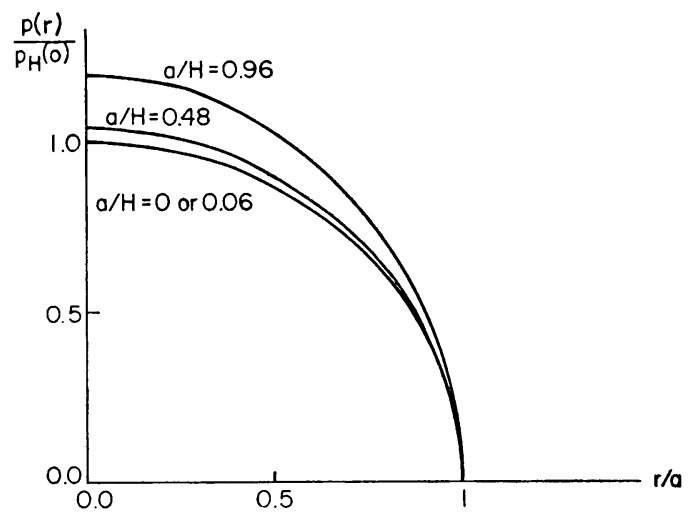

(a)

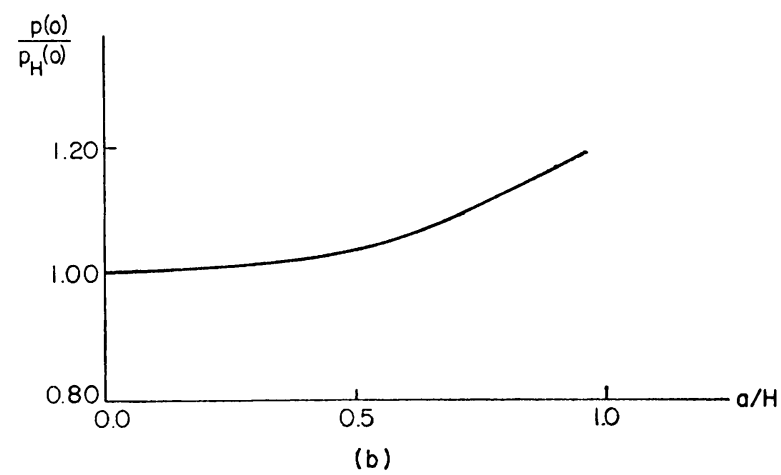

FIG. 1. (a). Normal contact stress distribution.

(b). Ratio of maximum contact stress. 


$$
\begin{aligned}
\sigma_{r r}= & \int_{0}^{\infty} s J_{0}(r s) \bar{p}(s) d s-(1-2 \nu) \int_{0}^{\infty} \frac{J_{1}(r s)}{r} \bar{p}(s) d s \\
& +\int_{0}^{\infty}\left[(1-\nu) J_{2}(s r)-(1+\nu) J_{0}(s r)\right] 2 H s^{2} \bar{p}(s) / F(2 H s) d s \\
= & p(r)-(1-2 \nu) \frac{1}{r^{2}} \int_{0}^{r} p(\lambda) \lambda d \lambda \\
& +\frac{1}{4 H^{2}} \int_{0}^{\infty}\left[(1-\nu) J_{2}\left(\frac{r}{2 H} u\right)-(1+\nu) J_{0}\left(\frac{u}{2 H} r\right)\right] u^{2} \bar{p}\left(\frac{u}{2 H}\right) / F(u) d u .
\end{aligned}
$$

Similarly, from (26) we have at $z=H$

$$
\begin{aligned}
\sigma_{\theta \theta}= & 2 \nu p(r)+(1-2 \nu) \frac{1}{r^{2}} \int_{0}^{r} p(\lambda) \lambda d \lambda \\
& -\frac{1}{4 H^{2}} \int_{0}^{\infty}\left[(1+\nu) J_{0}\left(\frac{u}{2 H} r\right)+(1-\nu) J_{2}\left(\frac{u}{2 H} r\right)\right] u^{2} \bar{p}\left(\frac{u}{2 H}\right) / F(u) d u .
\end{aligned}
$$

In (27) and (28) we may see that the first two terms are independent of $H$. They are actually the half-space solutions for the radial and circumferential surface stresses in terms of a general normal contact stress. However, the third terms which are functions of $H$ describe the effect that the thickness of the plate has on the surface stress distribution. This effect increases with decreasing thickness and vanishes when $H$ tends to infinity. If, in (27) and (28), we let $H \rightarrow \infty$ and $p(r)=p_{H}(r)$, we immediately obtain the surface stress distributions of a half-space subjected to a spherical indentation as given in [3]. In the half-space solution the surface stresses $\sigma_{r r}$ and $\sigma_{\theta \theta}$ are equal in magnitude but opposite in sign. However, this is no longer true on the surface of a plate, as seen from (27) and (28). Nevertheless the maximum tensile stress which occurs along the circle of contact area in a half-space still occurs along the same circle in the surface of a plate, as shown in Fig. 2(a). Figures 2(a), (b) were obtained as a result of the following considerations. If we recall the method for obtaining the contact stresses in the previous section, and if we let $r=a$ and $\lambda=a y$ in (27), we may make the assertion that the proportion between the surface stress $\sigma_{r r}(a)$ in a plate and ${ }_{H} \sigma_{r r}(a)$ in a half-space, i.e., $\sigma_{r r}(a) /{ }_{H} \sigma_{r r}(a)$, depends only on the Poisson ratio $\nu$ and the geometric parameter $a / H$. By varying the value of $a / H$ and taking $\nu=0.25$, the values of the ratio $\sigma_{r r}(a) /{ }_{H} \sigma_{r r}(a)$ were calculated to obtain the results shown in Fig. 2(a), (b). From this figure we may see that for sufficiently small $a / H$ the half-space solution is sufficiently accurate, but the ratio $\sigma_{r r}(a) /{ }_{H} \sigma_{r r}(a)$ becomes as large as 1.9 for $a / H=0.96$. In other words, if the indenter is sufficiently large and the plate is sufficiently thin, the maximum tensile stress in the plate is much higher than the corresponding value calculated from the half-space solution.

It should be pointed out here that (27) and (28) are true only for $r>0$. At $r=0$ the limit of the second terms in the equations are not defined. If, however, we write $J_{2}(s r)$ in terms of $J_{0}(s r)$ and $J_{1}(s r)$ in the first part of (27) and carry out the integration, we have at $z=H$ and $r=0$,

$$
\sigma_{r r}=\sigma_{\theta \theta}=\frac{1+2 \nu}{2} p(0)-\frac{1+\nu}{4 H^{2}} \int_{0}^{\infty} u^{2} \tilde{p}\left(\frac{u}{2 H}\right) / F(u) d u .
$$

The first term is again the same as the result given in [3]. 
There is also a significant point to be studied here. It was shown in [3] that tensile stress exists along the line $r=0$ inside the material of a half-space, but its magnitude is negligibly small. For a plate, however, it is found here by numerical calculation that the internal tensile stress exists along $r=0$ and reaches its maximum at $z=0$. Indeed we may find from (25) and (26) that along $r=0, \sigma_{r r}=\sigma_{\theta \theta}$ and at $z=r=0$

$\sigma_{\theta \theta}(0,0)=\sigma_{r r}(0,0)=-\frac{1}{4 H^{2}} \int_{0}^{\infty}\left[\frac{u}{2} \cosh \frac{u}{2}-(1+2 v) \sinh \frac{u}{2}\right] u \bar{p}\left(\frac{u}{2 H}\right) / F(u) d u$.

We may again show that the ratio $\sigma_{\theta \theta}(0,0) /{ }_{H} \sigma_{r r}(a)$ depends only on $\nu$ and $a / H$. For various values of $a / H$ and for $\nu=0.25$, (30) was evaluated and the results are shown also in Fig. 2(b). It may be seen there that for small $a / H, \sigma_{\theta \theta}(0,0) /{ }_{H} \sigma_{r r}(a)$ is small, as is to be expected, but it becomes as large as $30 \%$ for $a / H=0.96$. This significant increase in internal tensile stress as $H$ decreases is suggested as a possible reason for the occurrence of large internal cracks observed only at high impact velocities where a thin layer of material was stressed [4].

IV. Viscoelastic contact stress. The problem here is geometrically identical
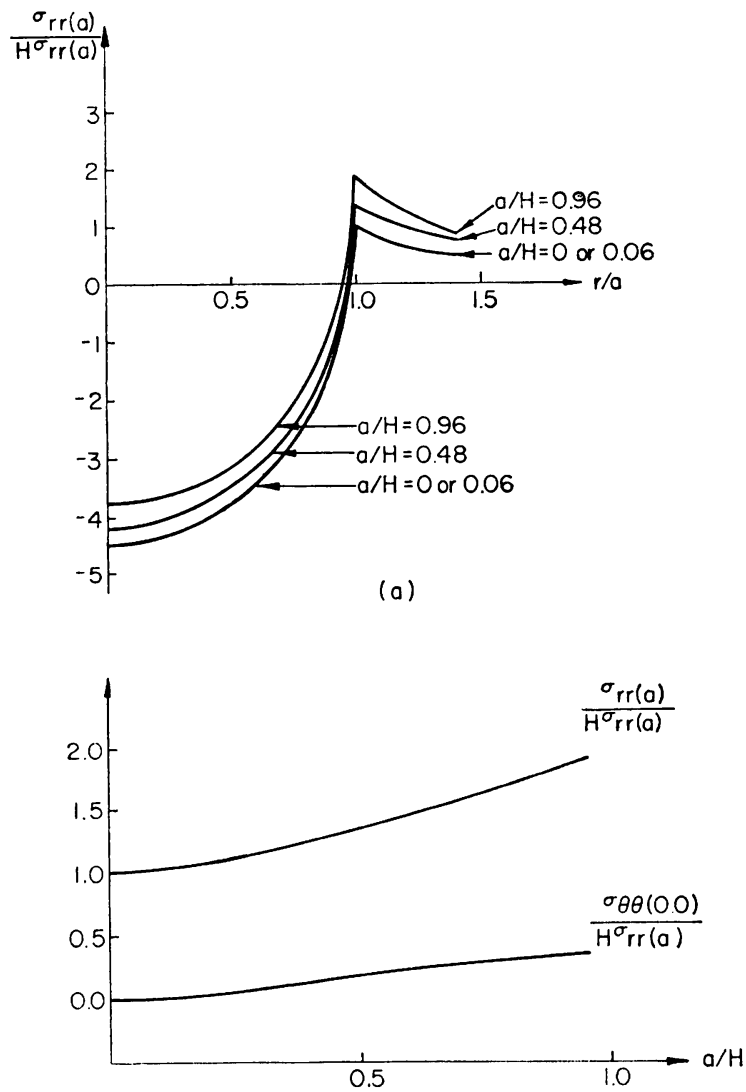

(b)

FIG. 2. (a). Radial surface stress distribution for $\nu=0.25$.

(b). Ratio of maximum radial surface stress for $\nu=0.25$ 
with that in Sec. 2. However, we use the following linear viscoelastic stress-strain relation [7], [8], [9]:

$$
\sigma_{i i}=\int_{0^{-}}^{t}\left[2 \mu(t-\tau) \frac{\partial}{\partial \tau} \epsilon_{i j}+\delta_{i j} \lambda(t-\tau) \frac{\partial}{\partial \tau} \epsilon_{k k}\right] d \tau
$$

and the boundary conditions at $z= \pm H$

$$
\begin{aligned}
\sigma_{z z} & =\sigma_{r z}=0 \text { for } r>a(t), \\
u_{z} & =g(r, t), \sigma_{r z}=0 \text { for } r \leq a(t) .
\end{aligned}
$$

In (31) $\mu(t)$ and $\lambda(t)$ are the relaxation moduli, and the lower limit $0^{-}$is used in order to take care of the possible jump in stresses and strains at $t=0$. The problem which was independent of time previously is now time-dependent, and it is solved here by the method of Laplace transform. If we define the Laplace transform

$$
p^{*}(b)=\int_{0^{-}}^{\infty} e^{-b t} p(t) d t
$$

the viscoelastic equation corresponding to (14) can be written as

$$
u_{z}^{*}(r, 0, b)=b \phi^{*}(b)\left[\int_{0}^{\infty} J_{0}(s r) \bar{p}_{v}^{*}(s, b) d s-\int_{0}^{\infty} Q(2 s H) J_{0}(s r) \bar{p}_{v}^{*}(s, b) d s\right]
$$

where

$$
b \phi^{*}(b)=\left(1-\nu^{*}\right) / b \mu^{*} .
$$

For convenience in inversion of the above transformed equations, we define the following new function:

$$
\psi^{*}(b)=1 / \phi^{*}(b)
$$

If $\nu(t)$ is a constant, $\psi(t)$ is of the same form as the relaxation modulus in shear $\mu(t)$, while $\phi(t)$ is of the same form as the creep compliance in shear. Eq. (34) can be inverted by using the method described in [7]. The method of inversion critically depends upon whether the radius of contact $a(t)$ is monotonically increasing or decreasing when the contact stresses $p_{v}(r, t)$ are calculated. We first consider the case of loading where $a(t)$ is monotonically increasing, varying from zero to its maximum $a\left(t_{m}\right)$. Before we invert (34) for $0 \leq t \leq t_{m}$, we consider the following inversion:

$$
\begin{aligned}
\bar{p}_{v}(s, t) & =\mathcal{L}^{-1}\left[b \phi^{*}(b) \bar{p}_{v}^{*}(s, b)\right] \\
& =\int_{0}^{\iota} \phi(t-\tau) \frac{\partial}{\partial \tau} \int_{0}^{a(\tau)} p_{v}(\lambda, \tau) \lambda J_{0}(\lambda s) d \lambda d \tau \\
& =\int_{0}^{a(t)} J_{0}(\lambda s) \lambda \int_{0}^{l} \phi(t-\tau) \frac{\partial}{\partial \tau} p_{v}(\lambda, \tau) d \tau d \lambda
\end{aligned}
$$

In view of the fact that $p_{v}(\lambda, \tau)=0$ for $\lambda \geq a(t)$, (37) is obtained by first replacing $a(\tau)$ by $a(t)$, and then interchanging differentiation and integration. If we now apply (37) to the inverse transform of (34), we have

$$
g(r, t)=\int_{0}^{\infty} J_{0}(s r) \bar{p}_{v}(s, t) d s-\int_{0}^{\infty} Q(2 H s) J_{0}(s r) \bar{p}_{v}(s, t) d s .
$$


If we consider $\int_{0}^{t} \phi(t-\tau)(\partial / \partial \tau) p_{v}(\lambda, \tau) d \tau$ as an unknown function, (38) is the same as (14) except for the constant $C$. Hence, the solution for the elastic contact stress may be used in connection with solving (38). Indeed, if the elastic contact stress for a given $a(t)$ is $p(r, t)$, the corresponding viscoelastic solution is

$$
\int_{0}^{t} \phi(t-\tau) \frac{\partial}{\partial \tau} p_{v}(r, \tau) d \tau=C p(r, t) .
$$

If we take the Laplace transform of (39) and use (36) and finally carry out the inversion, we have

$$
p_{v}(r, t)=C \int_{0^{-}}^{t} \psi(t-\tau) \frac{\partial}{\partial \tau} p(r, \tau) d \tau .
$$

Thus the viscoelastic contact stress is determined for the case of loading. Furthermore, if we examine the role of $C$ in (38) and (14), we may see from (17) and (39) that for elastic and viscoelastic solids with the same radius of contact $a(t)$ the depths of penetration $g(0, t)$ are identical. This result was also obtained in the half-space solution [7].

We have just shown that the method described in [7] is applicable here for the case of loading. For unloading in which $a(t)$ is assumed to be monotonically decreasing for $t_{m}<t<T$ as shown in Fig. 3, the method in [7] can also apply. In Fig. 3 we define $a\left(t^{\prime}\right)=a(t)$ for $t^{\prime}<t$. No detail is given here for unloading, but the contact stress may be found by the methods described in [7]:

$$
p_{v}(r, t)=\int_{0^{-}}^{t^{\prime \prime}(t)} \psi(t-\tau) \frac{\partial}{\partial \tau} C p(r, \tau) d \tau \text { for } t_{m}<t<T .
$$

Since $g(0, t)$ for loading was previously shown to be identical for elastic and viscoelastic plates, we may readily induce from [7] the following result for unloading:

$$
g_{v}(0, t)=g(0, t)-\int_{t_{m}}^{t} \phi(t-\tau) \frac{\partial}{\partial \tau} \int_{t^{\prime}(\tau)}^{\tau} \psi(\tau-\eta) \frac{\partial}{\partial \eta} g(0, \eta) d \eta d \tau
$$

where $g_{v}(0, t)$ and $g(0, t)$ are, respectively, the viscoelastic and elastic depths of penetration for $t_{m}<t<T$.

V. Discussion and conclusions. The contact stress set up by an arbitrary axisymmetrical indentation on the surface of an elastic plate resting on a rigid foundation was written as the sum of two components, namely the associated half-space solution and a thickness effect term. For spherical indentation the proportion of the latter term to the half-space term was shown to depend not on the material constants but on the ratio of the radius of contact $a$ to the thickness $H$. The thickness effect on the contact stress

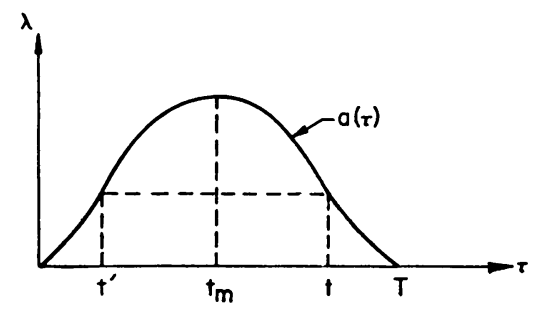

FIG. 3. Radius of contact between a rigid indenter and a viscoelastic solid. 
becomes increasingly important with decreasing thickness. For small value of $a / H$ the half-space solution is a sufficiently accurate approximation. This emphasizes the applicability of the Hertz solution in [1]. For $a / H=0.95$, however, the maximum plate contact stress in excess of the half-space solution is as large as $20 \%$. Contact stress distributions were calculated for various values of $a / H$, and the shapes of distribution set up by spherical indentation were seen not to deviate appreciably from each other.

The most remarkable result obtained here is perhaps the magnification of the maximum surface tensile stress due to the effect of the plate thickness. The radial and circumferential stresses in the surface of the plate were written as the sum of the stresses due to the thickness effect and the corresponding half-space stresses in terms of a general normal contact stress. For spherical indentation the maximum tensile stress was shown to occur radially along the circle of contact as in the half-space, and the ratio of the plate surface stresses to the corresponding half-space stresses depends not on the material properties, except for $\nu$, but rather on the geometrical parameter $a / H$. The value of this ratio increases with increasing $a / H$. For $a / H$ sufficiently small, the plate stresses tend to the corresponding half-space stresses. For $a / H=0.95$ the maximum plate tensile stress is twice as large as the corresponding half-space stress.

The contact stress in a viscoelastic plate under symmetrical rigid indentations was obtained and written in terms of the associated elastic solution and the viscoelastic constants of the material. The relation between the viscoelastic and the elastic contact stresses in the plate is the same as that in a half-space [7].

Acknowledgments. The author wishes to thank Professor H. J. Weiss for many useful discussions. The financial support from the Engineering Research Institute at the Iowa State University is also gratefully acknowledged.

\section{REFERENCES}

[1] Y. M. Tsai and H. Kolsky, A theoretical and experimental investigation of the flaw distribution on glass surfaces, J. Mech. Phys. Solids 15, 29-46 (1967)

[2] H. Hertz, Collected works, vol. 1, Leipzig Barth, 1882, p. 174

[3] M. T. Huber, Zur Theorie der Berührung fester elastischer Körper, Ann. Physik 14, 153-163 (1904)

[4] Y. M. Tsai and H. Kolsky, A study of the fractures produced in glass blocks by impact, J. Mech. Phys. Solids 15, 263-278 (1967)

[5] A. E. Green and W. Zerna, Theoretical elasticity, Clarendon Press, Oxford, 1960, p. 169

[6] H. Lamb, On Boussinesq's problem, Proc. London Math. Soc. 34, 276-284 (1902)

[7] T. C. T. Ting, The contact stresses between a rigid indenter and a viscoelastic half-space, J. Appl. Mech. Trans. ASME E33 4, 845-854 (1966)

[8] E. H. Lee and J. R. M. Radok, The contact problem for viscoelastic bodies, J. Appl. Mech. 27, 438-444 (1960)

[9] S. C. Hunter, The Hertz problem for a rigid spherical indenter and a viscoelastic half-space, J. Mech. Phys. Solids 8, 219-234 (1960) 